\title{
Cointegration of event-related potential (ERP) signals in experiments with different electromagnetic field (EMF) conditions
}

\author{
Argiro E. Maganioti ${ }^{1 *}$, Hountala D. Chrissanthi ${ }^{1}$, Papageorgiou C. Charalabos ${ }^{2,3}$, \\ Rabavilas D. Andreas ${ }^{3}$, Papadimitriou N. George ${ }^{2}$, Capsalis N. Christos ${ }^{1}$ \\ ${ }^{1}$ National Technical University of Athens, Department of Electrical Engineering, Division of Information Transmission Systems and \\ Material Technology, Athens, Greece; *Corresponding Author: roumag@mail.ntua.gr \\ ${ }^{2}$ Department of Psychiatry, Eginition Hospital, University of Athens, Athens, Greece \\ ${ }^{3}$ University Mental Health Research Institute (Umhri), Athens, Greece
}

Received 11 December 2009; revised 20 February 2010; accepted 23 February 2010.

\begin{abstract}
Due to their non-stationarity, ERP signals are difficult to study. The concept of cointegration might overcome this problem and allow for the study of the co-variability between whole ERP signals. In this context cointegration factor is defined as the ability of an ERP signal to co-vary with other ERP signals. The aim of the present study was to investigate whether the cointegration factor is dependent on different EMF conditions and gender, as well as the locations of the electrodes on the scalp. The findings revealed that women have a significantly higher cointegration factor than men, while all subjects have increased cointegration factors in the presence of EMF. The cointegration factor is location dependent, creating a distinct cluster of high cointegration capacity at the central and lateral electrodes of the scalp, in contrast to clusters of low cointegration capacity at the anterior and posterior electrodes There seem to be distinct similarities of the present findings with those from standard methodologies of the ERPs. In conclusion cointegration is a promising tool towards the study of functional interactions between different brain locations.
\end{abstract}

Keywords: EMF; ERP; Stationarity; Cointegration; ACF

\section{INTRODUCTION}

The electroencephalogram (EEG) is a non-invasive technique, providing a millisecond by millisecond readout of the brain's processing of information, and is relatively inexpensive to implement. Event-related potentials (ERPs) are a reflection of the brain's electrical response to stimulation. Typically, the event-related activity is small and is, thus difficult to view in the single trial. It is usually covered by the ongoing 'spontaneous' EEG. ERPs techniques overcome this initially poor signal to noise ratio by averaging across many trials, typically from about 15 to several hundred [1].

Both EEG and ERP signals are time series. Stationary EEG signals are successfully analyzed in the frequency domain using Fourier transformations [2]. It has been found that electromagnetic fields (EMF), similar to those emitted by mobile phones, have a gender specific effect on the energy of the EEG [3]. However, Fourier transformations cannot be applied on the non-stationary ERP signals. There are a number of alternative approaches that overcome the issue of non-stationarity, such as windowed Fourier $[4,5]$ and wavelet analysis $[6,7]$.

The majority of the studies analysing ERPs focuses on certain components of the ERP signal (P50, N100, P200, N200, P300, N400, P600) [8,9], each of which receives a particular functional interpretation in the physiology of the brain. Very few analyses have been made employing the whole time-series and even less regarding the correlation among the activity of different electrodes. The common methods used for processing ERPs include coherence [10], regression [11], correlation [12] and Granger causality [13]. Most of these methods were first developed in economic sciences.

In the present paper the whole series of the ERP is employed as a unit and its non-stationarity is taken into consideration. The approach, based on a concept introduced by Granger in analysing economic time series, is cointegration [14]. With regards to brain stimuli, cointegration has been used for linear autoregressive EEG modeling [15] and for utilizing the stationarity of the EEG multivariate time series (MTS) [16]. 
In the present study, cointegration is defined as the ability of two ERP signals to co-vary in time. The aim is to investigate whether this ability is dependent on different EMF conditions and gender, as well as the locations of the electrodes on the scalp.

\section{METHODS}

\subsection{Participants}

Two different groups of people, who took part in two separate experiments, were used in this study. The first group consisted of nineteen healthy individuals ( 9 men and 10 women, mean age $=23.3 \pm 2.23$ years, mean education $=16.9 \pm 1.82$ years) and participated in the first experiment. The participants of the second experiment were twenty healthy individuals (10 men and 10 women, mean age $=22.75 \pm 2.71$ years, mean education $=16.3 \pm 1.71$ years $).$ In both experiments, the male and female subgroups were homogeneous with regards to age and educational level. All participants were righthanded and had no history of any hearing problem. Informed consent was obtained from all subjects.

\subsection{Experimental Setup and Measurement Procedure}

The two experiments were in fact the same as far as the evaluation method is concerned. The subjects were evaluated with the digit span Wechsler Auditory test [17]. A warning stimulus of either high $(3000 \mathrm{~Hz})$ or low frequency $(500 \mathrm{~Hz})$ was presented through earphones to the subjects, who were asked to memorize the numbers that followed. The warning stimulus lasted $100 \mathrm{msec}$. A one second interval followed the onset of the warning stimulus and then the numbers to be memorized were presented by a male voice. At the end of the number sequence presentation, the same signal tone was repeated. The signals were recorded for a $1500 \mathrm{msec}$ interval, divided into $500 \mathrm{msec}$ before the warning stimulus (EEG) and 1000 msec after that (ERP) [3]. The numbers were recalled by the subject in the same (low frequency tone) or in the opposite order (high frequency tone) than that presented to the participant.

The total task consisted of 52 repetitions for a period of about $45 \mathrm{~min}$. The subjects performed the tasks twice, with and without radiation, with an interval of two weeks between the measurements. The order in which the subject was exposed at the EMF (exposure at the first or second visit) was random and the subjects were unaware of the experimental condition.

The only difference between the two experiments was the frequency of the EMF signal at which the subjects were exposed. The first experiment involved an antenna emitting $900 \mathrm{MHz}$ electromagnetic field, with mean power at $64 \mathrm{mWatt}$, while in the second one the antenna used emitted $1800 \mathrm{MHz}$ electromagnetic field, with mean power at $128 \mathrm{mWatt}$. In both experiments the signal was not modulated.

The experimental setup was the same in both cases and included a Faraday room, which screened any electromagnetic interference that could affect the measurements. The subjects sat in an anatomical chair and a certified dipole antenna was fixed near their right ear. Care was taken so that the distance between telephone and ear (about $20 \mathrm{~cm}$ ) was constant during the whole session. The antenna was driven by a signal generator, which could be switched on or off.

The electrophysiological signals were recorded with $\mathrm{Ag} / \mathrm{AgCl}$ electrodes. Electrode resistance was kept constantly below $5 \mathrm{k} \Omega$. EEG activity was recorded from 15 scalp electrodes (Fp1, F3, C5, C3, Fp2, F4, C6, C4, O1, $\mathrm{O} 2, \mathrm{P} 4, \mathrm{P} 3, \mathrm{Pz}, \mathrm{Cz}, \mathrm{Fz}$ ) based on the International 10-20 system of Electroencephalography [18], referred to both earlobes. An electrode placed on the subject's forehead served as ground. The bandwidth of the amplifiers was set at $0.05 \mathrm{~Hz}$ to $35 \mathrm{~Hz}$. During the administration of stimuli, the subjects had their eyes closed in order to minimize eye movements and blinks. Eye movements were recorded through electro-oculogram (EOG) and recordings with EEG higher than $75 \mu \mathrm{V}$ were rejected which on the average were $2.1 \pm 1.4$ trials from the total of 52. Warning stimuli, as well as the numbers to recall were presented binaurally via earphones at an intensity of $65 \mathrm{~dB}$ sound pressure level. The earphones did not have metal components in order to avoid EMF concentration. The evoked biopotential signal was submitted to an analogue-to-digital conversion, at a sampling rate of 1 $\mathrm{KHz}$.

\subsection{Data Transformation}

For each question 1500 data points, each corresponding to time segments of $1 \mathrm{msec}$ duration for each electrode were saved. In order to maximize the signal to noise ratio for each subject and each channel all values were average referenced on the basis of the grand average across the 52 repetitions of the EEG values. This procedure was done separately for each EMF condition in both experiments. Artifact-contaminated epochs with a signal deviation of $>75 \mu \mathrm{V}$ in the EEG or $100 \mu \mathrm{V}$ in the EOG were excluded. The final data for analysis for each subject and condition consists of 1500 amplitude values for each electrode, expressed in $\mu$ Volts corresponding to the $1500 \mathrm{msec}$ of the time period [3].

A representative chart of the primary recordings of the amplitude values are shown in Figure 1.

\subsection{Stationarity and Integration}

A strict stationary process is a stochastic process whose probability distribution does not vary over time; basic characteristics such as the mean $\left(E\left(x_{t}\right)\right)$ and the vari- 


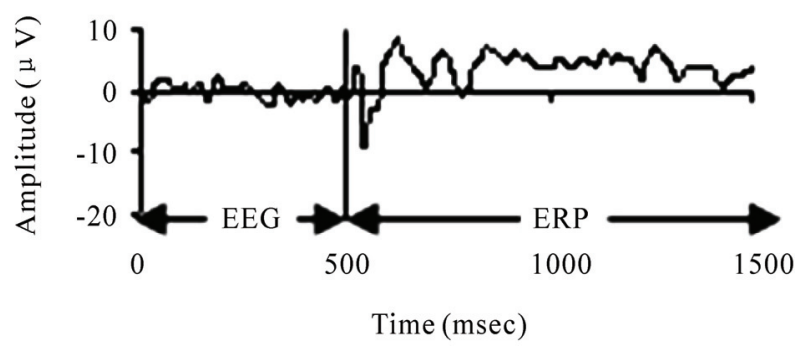

Figure 1. Representative graph of the 1500 amplitude values of the electrode Fz.

ance $\left(\operatorname{var}\left(x_{t}\right)\right)$ remain constant over time. Stationary time series are easier to analyze and forecast, therefore non-stationary raw data are often transformed, in order to become stationary. Non-stationary processes contain a trend. Trend represents a general systematic linear or non-linear component that changes over time and does not repeat, or at least does not repeat within the time range captured by the data. There are two kinds of trend; deterministic and stochastic. Time series with deterministic trend have constant variance but non-constant mean, whereas the ones with stochastic trend exhibit non-constant variance. Some processes may contain both stochastic and deterministic trends, which means that they combine a random walk $\left(r_{t}\right)$ and a deterministic trend $(\beta t)$, plus an error term.

$$
x_{t}=r_{t}+\beta t+\varepsilon_{t}
$$

A non-stationary process with deterministic trend is transformed into a stationary one by regressing it on time $t$. The most common method for removing stochastic trends from a non-stationary process is differencing. Differencing of a time series $x_{t}$ in discrete time $\mathrm{t}$ is the transformation of the series $x_{t}$ to a new time series $\operatorname{dif}_{t}$ where the values $\operatorname{dif}_{t}$ are the differences between consecutive values of $x_{t}$. The $d_{t h}$ differences of a time series are described by the following expression:

$$
\operatorname{dif}_{t}^{(d)}=\operatorname{dif}_{t}^{(d-1)}-\operatorname{dif}_{t-1}^{(d-1)},
$$

where the top index $d$ means the order of the difference.

Many time series need to be differenced more than once in order to achieve stationarity. From this comes the definition of integration: a time series is said to be integrated of order $d$, in short, I(d), if it becomes stationary after differencing $d$ times. A series which is $\mathrm{I}(\mathrm{d})$ is also said to have $d$ unit roots.

\subsection{Testing for Stationarity: KPSS Test}

The KPSS test [20] is commonly used to test for stationnarity in time-series data. Let $\left\{x_{t}\right\}, t=1,2, \ldots, N$, be the observed series for which we wish to test stationarity. Assume that the series can be decomposed into the sum of a deterministic trend, a random walk, and a stationary error with the following linear regression model

$$
x_{t}=r_{t}+\beta t+\varepsilon_{t}
$$

where $r_{t}$ is a random walk, i.e., $r_{t}=r_{t-1}+u_{t}$ and $u_{t}$ is independent identically distributed (iid) $N\left(0, \sigma_{u}{ }^{2}\right), \beta t$ is a deterministic trend and $\varepsilon_{t}$ is a stationary error.

To test in this model if $x_{t}$ is a stationary process, the null hypothesis will be $\sigma_{u}{ }^{2}=0$, which means that the intercept is a fixed element, against the alternative of a positive $\sigma_{u}{ }^{2}$. Under the null hypothesis, in the case of stationarity, the residuals $e_{t}(t=1,2, \ldots, N)$ are from the regression of $x$ on an intercept and time trend, $e_{t}=\varepsilon_{t}$. Let the partial sum process of the $e_{t}$ be

$$
S_{t}=\sum_{j=1}^{t} e_{j},
$$

and $\sigma^{2}$ be the long-run variance of $e_{t}$, which is defined as

$$
\sigma^{2}=\lim N^{-1} E\left[S_{N}^{2}\right] .
$$

The consistent estimator of $\sigma^{2}$ can be constructed from the residuals $e_{t}$ by the equation below [19]

$$
\hat{\sigma}^{2}(p)=\frac{1}{N} \sum_{t=1}^{N} e_{t}^{2}+\frac{2}{N} \sum_{j=1}^{p} w_{j}(p) \sum_{t=j+1}^{N} e_{t} e_{t-j},
$$

where $p$ is the truncation $\operatorname{lag}, w_{j}(p)$ is an optional weighting function that corresponds to the choice of a special window, e.g., Bartlett window (Bartlett, 1950) $w_{j}(p)=1-j /(p+1)$.

Then the KPSS test statistic is given by

$$
K P S S=N^{-2} \sum_{t=1}^{N} S_{t}^{2} / \hat{\sigma}^{2}(p) .
$$

Under the null hypothesis of stationarity,

$$
K P S S \rightarrow \int_{0}^{1} V_{2}(r)^{2} d r,
$$

where $V_{2}(r)$ is the second level Brownian bridge, given by

$$
V_{2}(r)=B(r)+\left(2 r-3 r^{2}\right) B(1)+\left(-6 r+6 r^{2}\right) \int_{0}^{1} B(s) d s .
$$

The upper tail critical values of the asymptotic distribution of the KPSS statistic are given by Kwiatkowski et al. [20]. It has been shown that KPSS test is the most powerful test for the stationarity of time series [20,21], therefore it will used in the present paper.

Further details of the test are given in Zivot and Wang (2002).

\subsection{Cointegration}

Granger and Newbold [22] have proven that using two I(1) time series, an apparently significant regression and correlation can be obtained, even if the two time series are independent. These regression results were coined as 
"spurious regressions".

In order to overcome this limitation, Granger introduced a new method, based on the notion that a linear combination between a pair of non-stationary integrated series can be stationary. This property is known as cointegration [14]. Cointegration is a formulation of the phenomenon that non-stationary integrated series can have linear combinations that have a lower degree of integration than the original series.

In this paper, the tool used for testing the co-variability of different electrodes is cointegration. The method of cointegration involves testing whether the residuals from a cointegrating regression are stationary. Consider two time series $\mathrm{y}_{1 t}$ and $\mathrm{y}_{2 t}$ which are both $\mathrm{I}(\mathrm{d})$. In general, any linear combination of $\mathrm{y}_{1 t}$ and $\mathrm{y}_{2 t}$ will be also $\mathrm{I}(\mathrm{d})$. However, if there exists a vector $(1,-\beta)$ ', such that the linear combination

$$
z_{t}=y_{1 t}-a-\beta \cdot y_{2 t}
$$

is $\mathrm{I}(\mathrm{d}-\mathrm{b}), \mathrm{d} \geq \mathrm{b}>0$, then, following Engle and Granger (1987), $y_{1 t}$ and $y_{2 t}$ are defined as cointegrated of order (d, b) denoted $y_{t}=\left(y_{1 t}, y_{2 t}\right)^{\prime} \sim \mathrm{CI}(\mathrm{d}, \mathrm{b})$, with $(1,-\beta)^{\prime}$ called the cointegrating vector [23].

\subsection{Data Processing}

In this study, the ERP signals are being analyzed in terms of cointegration. More precisely, the stationarity of the ERPs of each electrode, for all the subjects, is being examined using the KPSS test. If the ERPs are found to be non-stationary (which is the case), they are properly differenced. This procedure is repeated until KPSS test indicates stationarity. In order to achieve stationarity the signals were differenced twice or three times. The null hypothesis of stationarity with upper tail critical value of the asymptotic distribution of the KPSS statistic 0.119 is tested.

Cointegration is tested for those electrodes that are integrated of the same order. The two ERPs are being regressed one against the other and the order of integration or stationarity of the residuals is tested. If the order of integration of the residuals is less than that of the two ERP signals then cointegration exists between the two variables. A $15 \times 15$ array is created for each subject in each condition and if cointegration exists between electrode $\mathrm{i}$ and electrode $\mathrm{j}$-provided that the two electrodes are integrated of the same order - 1 is placed at the $[i, j]$ cell. Else, if no such relationship exists, a 0 is placed in the cell. For each electrode, the respective column is summed, and the result is a $1 \times 15$ vector, containing the exact number of cointegrations for each electrode for the specific subject. This number is subsequently normalized by dividing by the maximum number of possible cointegrations (in this case 14). This number is called Cointegration Factor $(\mathrm{CF})$ of the electrodes. According to the above, the values of the electrode $\mathrm{CF}$ can range from 0 to 1 . In the same way, the mean value of electrode CFs is the Aggregate Cointegration Factor (ACF).

\section{STATISTICAL ANALYSIS}

The aggregate CF was subjected to two-way ANOVA with gender (male, female) and EMF condition (none, $900 \mathrm{MHz}, 1800 \mathrm{MHz}$ ) as the independent factors. Likewise the CFs at the fifteen electrodes were subjected to MANOVA with the same independent factors. Finally, in order to examine whether the CF differs among different electrodes (locations), an ANOVA procedure with repeated measures was performed for all the subjects and measurements. The statistical significance was set at 0.05 .

\section{RESULTS}

Univariate analysis of variance with ACF as the dependent variable and EMF condition (off, $900 \mathrm{MHz}, 1800 \mathrm{MHz}$ ), gender (male, female) and their interaction as the independent factors revealed a significant EMF effect $\left(\mathrm{F}_{2,77}=\right.$ $4, p=0.022)$ as well as a significant gender effect $\left(F_{1,77}\right.$ $=4, p=0.048$ ), but no interaction effect. Figure 2 helps to clarify the direction of the differences between EMF conditions and genders. As post-hoc comparisons with Bonferroni corrections show, women have in general a significantly higher ACF than men $(p=0.048)$. The presence of radiation increases ACF. As a result ACF in the presence of $1800 \mathrm{MHz}$ is significantly higher than in the absence of radiation $(p=0.029)$.

In order to further qualify the effect of EMF and gender on $\mathrm{CF}$ for each electrode individually, the CFs of the 15 electrodes were subjected to MANOVA with EMF condition (off, $900 \mathrm{MHz}, 1800 \mathrm{MHz}$ ), gender (male, female) and their interaction as independent factors. Table 1 shows the significance of their effects on the CF for all the electrodes. Significant effects are shown in bold. Once again the variability of the CF of the leads is mostly due to the effect of EMF conditions and to a lesser degree of gender differences, while the EMF $\mathrm{x}$ Gender interaction does not have any significant effect. These effects were more obvious at F3, C5, C3, C6, C4, $\mathrm{O} 1, \mathrm{P} 4, \mathrm{Pz}$ and $\mathrm{Cz}$.

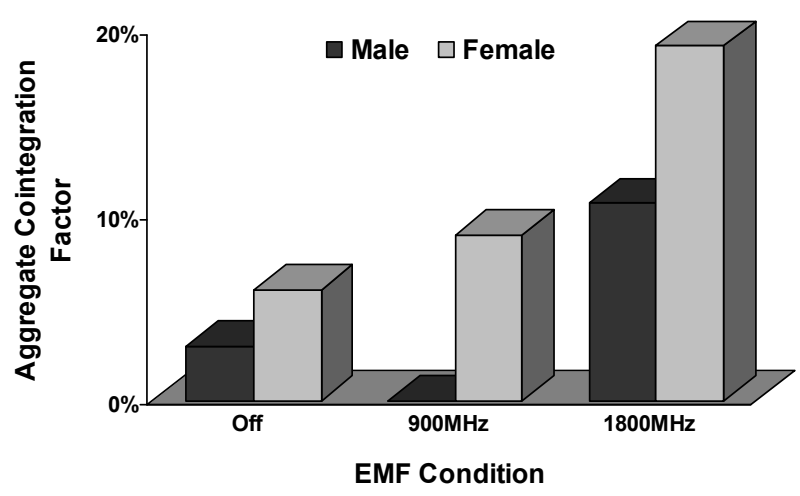

Figure 2. Average of the Aggregate Cointegration Factor for each gender and EMF condition. 
Table 1. Significance of the effects of EMF condition and gender on the cointegration factor of the electrodes.

\begin{tabular}{cccc}
\hline & EMF & Gender & EMF x Gender \\
\hline Lead & p-value & p-value & p-value \\
Fp1 & 0.445 & 0.302 & 0.300 \\
F3 & $\mathbf{0 . 0 3 6}$ & $\mathbf{0 . 0 4 4}$ & 0.430 \\
C5 & $\mathbf{0 . 0 0 0}$ & $\mathbf{0 . 0 3 5}$ & 0.218 \\
C3 & $\mathbf{0 . 0 2 3}$ & $\mathbf{0 . 0 1 1}$ & 0.517 \\
Fp2 & 0.561 & 0.174 & 0.709 \\
F4 & 0.174 & 0.114 & 0.346 \\
C6 & $\mathbf{0 . 0 4 5}$ & 0.200 & 0.624 \\
C4 & $\mathbf{0 . 0 0 5}$ & $\mathbf{0 . 0 0 8}$ & 0.676 \\
O1 & $\mathbf{0 . 0 2 3}$ & 0.546 & 0.629 \\
O2 & 0.457 & 0.142 & 0.516 \\
P4 & $\mathbf{0 . 0 3 5}$ & 0.505 & 0.936 \\
P3 & 0.067 & 0.130 & 0.435 \\
Pz & $\mathbf{0 . 0 1 7}$ & 0.295 & 0.775 \\
Cz & $\mathbf{0 . 0 3 6}$ & $\mathbf{0 . 0 2 7}$ & 0.503 \\
Fz & 0.249 & 0.102 & 0.684 \\
\hline
\end{tabular}

The repeated ANOVA procedure with the CFs at the fifteen electrodes as the within subjects factor proved that there exist significant differences between the means of the CFs at different locations $\left(\mathrm{F}_{14,77}=3.7, \mathrm{p}<0.001\right)$.

As Figure 3 shows high CFs seem to cluster in the central and lateral electrodes, while electrodes with low CFs are grouped mainly in the posterior but also the anterior electrodes.

\section{DISCUSSION}

As Granger notes, "cointegration signifies co-movements among trending variables which can be exploited to test for the existence of equilibrium relationships within a fully dynamic specification framework" [23]. In the present study, the cointegration factor was defined as the ability of ERP signals to co-vary in time. Results showed that the values of the CFs seem to follow specific patterns forming distinct clusters, which discriminate the central and lateral electrodes, having relatively high CFs, from the anterior and posterior ones. Furthermore, women have a significantly higher CF than men, while all subjects have increased CFs in the presence of EMF.

The above findings seem to be in congruence with other findings regarding typical characteristics of the ERP signal. Specifically, with regards to EMF effects, EEG studies showed an increase of spectral power in the

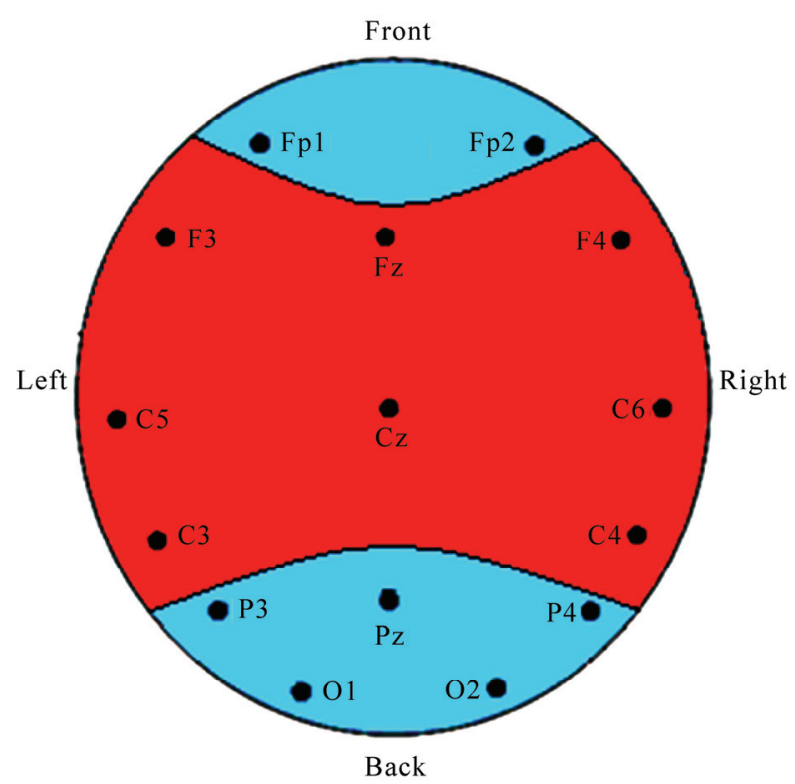

Figure 3. Mean values of the cointegration factors at different locations on the scalp. Red area signifies cointegration factors greater than $7.5 \%$, while the blue areas contain electrodes with cointegration factors less than $7.5 \%$.

alpha band [2,24,25], while ERP studies have demonstrated reduced N100 amplitudes, shortened N100 latencies and prolonged P300 latencies [26]. In this context, it has been shown that EMF modulated the event-related desynchronization/synchronization (ERD/ERS) responses in the approximately 4-8 Hz EEG frequencies [27]. In view of these collected observations the authors concluded that EMF could influence brain activity through thermal and non-thermal mechanisms [28,29].

However, a number of studies failed to find EMF effects upon brain physiology [30,31]. This might be attributed to the fact that these studies focused not on the whole ERP recording, but on certain components of ERP. In contrast, the present study analyzed the total ERP signal, specifically its cointegration capacity, which is likely to represent a more valid picture of the effect of EMF exposure on the ERP.

The gender-related differences of CF may be related to different strategies activated due to sex-related functional brain organization, as indicated from psychophysiological and neurobiological studies [32-35]. There also appears to be consistent evidence that EEG coherence varies systematically with gender [36].

Finally, the auditory nature of the warning stimuli that elicit the ERPs might be the possible reason that affects the ERP activity in the temporo-parietal region $[37,38]$ creating the distinct clusters of CF presently found.

To the best of our knowledge, this is the first attempt to apply the concept of cointegration in the study of co-variability of ERP signals. Cointegration seems to be a promising tool towards the study of functional interact- 
tions between different brain locations. Also, the method can be applied on EEG data, obtained from different clinical and technical experimental conditions. Finally, it is our immediate object to demonstrate the feasibility of the cointegration method on additional cogntive tasks that involves activities in the frontal and occipital scalp locations, using data from ongoing experiments.

\section{ACKNOWLEDGEMENTS}

The authors would like to thank M. Kyprianou, Scientific Investigator, Athens, Greece, for his support on the statistical analysis of the experimental results.

\section{REFERENCES}

[1] Fabiani, M., Gratton, G. and Coles, M. (2000) Eventrelated potentials: Methods, theory and applications. Handbook of Psychophysiology, 3rd Edition. Cambridge University Press, New York.

[2] Laufs, H., Kleinschmidt, A., Beyerle, A., Eger, E., SalekHaddadi, A., Preibisch, C. and Krakow, K. (2003) EEGcorrelated fMRI of human alpha activity. NeuroImage, 19(4), 1463-1476.

[3] Papageorgiou, C.C., Nanou, E.D., Tsiafakis, V.G., Capsalis, C.N. and Rabavilas, A.D. (2004) Gender related differences on the EEG during a simulated mobile Phone signal. Neuroreport, 15(16), 2557-2560.

[4] Agam, Y. and Sekuler, R. (2007) Interactions between working memory and visual perception: An ERP/EEG study. NeuroImage, 36(3), 933-942.

[5] Edwards, E., Soltani, M., Deouell, L.Y., Berger, M.S. and Knight, R.T. (2005) High gamma activity in response to deviant auditory stimuli recorded directly from human cortex. Journal of Neurophysiology, 94, 4269-4280.

[6] Yordanova, J., Rosso, O.A. and Kolev, V. (2003) A transient dominance of theta event-related brain potential component characterizes stimulus processing in an auditory oddball task. Clinical Neurophysiology, 114(3), 529540 .

[7] Quiroga, R.Q., Sakowitz, O.W., Basar, E. and Schürmann, M. (2001) Wavelet Transform in the analysis of the frequency composition of evoked potentials. Brain Research Protocols, 8(1), 16-24.

[8] Chlubnová, J., Kremláček, J., Kubová, Z. and Kuba, M. (2005) Visual evoked potentials and event related potentials in congenitally deaf subjects. Physiological Research, 54, 577-583.

[9] Sanders, L.D., Newport, E.L. and Neville, H.J. (2002) Segmenting nonsense: An event-related potential index of perceived onsets in continuous speech. Nature Neuroscience, $\mathbf{5}, 700-703$.

[10] Shibata, T., Shimoyama, I., Ito, T., Abla, D., Iwasa, H., Koseki, K., Yamanouchi, N., Sato, T. and Nakajima, Y. (1998) The synchronization between brain areas under motor inhibition process in humans estimated by eventrelated EEG coherence. Neuroscience Research, 31(4), 265-271.

[11] Hauk, O., Davis, M.H., Ford, M., Pulvermüller, F. and Marslen-Wilson, W.D. (2006) The time course of visual word recognition as revealed by linear regression analysis of ERP data. NeuroImage, 30(4), 1383-1400.

[12] Nikolaev, A.R., Ivanitsky, G.A., Ivanitsky, A.M., Posner, M.I. and Abdullaev, Y.G. (2001) Correlation of brain rhythms between frontal and left temporal (Wernicke's) cortical areas during verbal thinking. Neuroscience Letters, 298(2), 107-110.

[13] Oya, H., Poon, P.W.F., Brugge, J.F., Reale, R.A., Kawasaki, H., Volkov, I.O. and Howard III, M.A. (2007) Functional connections between auditory cortical fields in humans revealed by Granger causality analysis of intra-cranial evoked potentials to sounds: Comparison of two methods. Biosystems, 89, 198-207.

[14] Engle, R. and Granger, C.W. (1987) Co-integration and error correction representation estimation and testing. Econometrica, 55(2), 251-276.

[15] Yang, K. and Shahabi, C. (2005) On the stationarity of multivariate time series for correlation-based data analysis. Proceedings of the Fifth IEEE International Conference on Data Mining, 805-808.

[16] Brandt, M.E., Ademoglu, A. and Pritchard, W.S. (2000) Non-linear prediction and complexity of alpha EEG activity. International Journal of Bifurcation and Chaos, 10(1), 123-133.

[17] Wechsler, D. (1955) Manual for the Wechsler adult intelligence scale. Psychological Corporation, New York.

[18] Jasper, H.H. (1958) The ten-twenty electrode system of the international federation. Electroencephalography and Clinical Neurophysiology, 10, 371-375.

[19] Newey, W.K. and West, K.D. (1987) A simple, positive semi-definite, heteroskedasticity and autocorrelation consistent covariance matrix. Econometrica, 55(3), 703-708.

[20] Kwiatkowski, D., Phillips, P.C.B., Schmidt, P. and Shin, Y. (1991) Testing the null hypothesis of stationarity against the alternative of a unit root. How sure are we that economic time series have a unit root? Journal of econometrics, 54, 158-178.

[21] Hobijn, B., Franses, P.H. and Ooms, M. (2004) Generalizations of the KPSS-test for stationarity. Statistica Neerlandica, 58(4), 483-502.

[22] Granger, C.W. and Newbold, P. (1974) Spurious regression in econometrics. Journal of Econometrics, 2(2), 111120.

[23] Dolado, J.J., Gonzalo, J. and Marmol, F. (2009) A Companion to Theoretical Econometrics, In: Baltagi, B.H., Ed., Blackwell Reference Online, 634-654

http://www.blackwellreference.com/public/book?id=g97 814051067649781405106764

[24] Croft, R.J., Chandler, J.S., Burgess, A.P., Barry, R.J., Williams, J.D. and Clarke, A.R. (2002) Acute Mobile phone operation affects neural function in humans. Clinical Neurophysiology, 113(10), 1623-1632.

[25] Curcio, G., Ferrara, M., Moroni, F., D'Inzeo, G., Bertini, M. and De Gennaro, L. (2005) Is the brain influenced by a phone call? An EEG study of resting wakefulness. Neuroscience Research, 53(3), 265-270.

[26] Hamblin, D.L., Wood, A.W., Croft, R.J. and Stough, C. (2004) Examining the effects of electromagnetic fields emitted by GSM mobile phones on human event-related potentials and performance during an auditory task. Clinical Neurophysiology, 115(1), 171-178.

[27] Krause, C.M., Björnberg, C.H., Pesonen, M., Hulten, A., 
Liesivuori, T., Koivisto, M., Revonsuo, A., Laine, M. and Hämäläinen, H. (2006) Mobile phone effects on children's event-related oscillatory EEG during an auditory memory task. International Journal of Radiation Biology, 82(6), 443-450.

[28] Challis, L.J. (2005) Mechanisms for interaction between RF fields and biological tissue. Bioelectromagnetics, Supplement 7 Review, 26, 98-106.

[29] Valentini, E., Curcio, G., Moroni, F., Ferrara, M., De Gennaro, L. and Bertini, M. (2007) Neurophysiological effects of mobile phone electromagnetic fields on humans: A comprehensive review. Bioelectromagnetics, 28, 415-432.

[30] Hamblin, D.L., Croft, R.J., Wood, A.W., Stough, C. and Spong, J. (2006) The sensitivity of human event-related potentials and reaction time to mobile phone emitted electromagnetic fields. Bioelectromagnetics, 27, 265-273.

[31] Yuasa, K., Arai, N., Okabe, S., Tarusawa, Y., Nojima, T., Hanajima, R., Terao, Y. and Ugawa, Y., (2006) Effects of thirty minutes mobile phone use on the human sensory cortex. Clinical Neurophysiology, 117(4), 900-905.

[32] Skrandies, W., Reik, P. and Kunze, C. (1999) Topography of evoked brain activity during mentalarithmetic and language tasks: sex differences. Neuropsychologia, 37(4), 421-430.

[33] Briere, M.E., Forest, G., Chouinard, S. and Godbout, R.
(2003) Evening and morning EEG differences between young men and women adults. Brain and Cognition, 53(2), 145-148.

[34] Dimpfel, W., Wedekind, W. and Keplinger, I. (2003) Gender difference in electrical brain activity during presentation of various film excerpts with different emotional content. European Journal of Medical Research, 8(5), 192-198.

[35] Razumnikova, O.M. and Vol'f, N.V. (2007) Gender differences in interhemisphere interactions during distributed and directed attention. Neuroscience and Behavioral Physiology, 37(5), 429-434.

[36] Barry, R.J., Clarke, A.R., McCarthy, R., Selikowitz, M., Johnstone, S.J. and Rushby, J.A. (2004) Age and effects in EEG coherence: I. Developmental trends in normal children. Clinical Neurophysiology, 115(10), 2252-2258.

[37] Haarala, C., Aalto, S., Hautzel, H., Julkunen, L., Rinne, J.O., Laine, M., Krause, B. and Hämäläinen, H. (2003) Effects of a $902 \mathrm{MHz}$ mobile phone on cerebral blood flow in humans: a PET study. Neuroreport, 14(16), 20192023.

[38] Cinel, C., Boldini, A., Russo, R. and Fox, E. (2007) Effects of mobile phone electromagnetic fields on an auditory order threshold task. Bioelectromagnetics, 28(6), 493496. 